\title{
High-latitude GPS phase scintillation and cycle slips during high-speed solar wind streams and interplanetary coronal mass ejections: a superposed epoch analysis
}

\author{
Paul Prikryl ${ }^{1 *}$, P Thayyil Jayachandran², Sajan C Mushini ${ }^{3}$ and lan G Richardson ${ }^{4,5}$
}

\begin{abstract}
Results of a superposed epoch (SPE) analysis of occurrence of phase scintillation and cycle slips at high latitudes keyed by arrival times of high-speed solar wind streams (HSS) and interplanetary coronal mass ejections (ICME) for years 2008 to 2012 are presented. Phase scintillation index $\sigma_{\Phi}$ is obtained in real time from L1 signal recorded at the rate of $50 \mathrm{~Hz}$ by specialized global positioning system (GPS) ionospheric scintillation and total electron content (TEC) monitors (GISTMs) deployed as a part of the Canadian High Arctic lonospheric Network (CHAIN). The phase scintillation, mapped as a function of magnetic latitude and magnetic local time, occurs predominantly on the dayside in the cusp and in the nightside auroral oval. The scintillation occurrence peaks on days of HSS or ICME impacts at the Earth's magnetosphere and tapers off a few days later, which is similar to day-to-day variability of geomagnetic activity and riometer absorption at high latitudes. ICMEs that are identified as magnetic clouds are significantly more geoeffective than HSSs and ICMEs with no or weak magnetic cloud characteristics. On their arrival day, magnetic clouds result in higher occurrence, and thus probability, of scintillation in the nightside auroral zone. The SPE analysis results are used to obtain cumulative probability distribution functions for the phase scintillation occurrence that can be employed in probabilistic forecast of phase scintillation at high latitudes.
\end{abstract}

Keywords: Ionosphere; Ionospheric irregularities; GPS scintillation; Solar wind disturbances; Space weather forecasting

\section{Background}

Ionospheric scintillation (Aarons 1982, 1997; Aarons et al. 2000; Basu et al. 1987, 1995, 1998) of the Global Navigation Satellite Systems (GNSS) signal severely degrades positional accuracy, causes cycle slips which can lead to loss of lock, and affects performance of radio communications and navigation systems. GNSS receiver tracking performance during severe scintillation conditions can be assessed by the analysis of receiver phaselocked-loop (PLL) jitter (Conker et al. 2003; Sreeja et al. 2011). At high latitudes, statistical characterization and climatology of scintillation of global positioning system (GPS) signals show prevalence of phase over amplitude

\footnotetext{
* Correspondence: paul.prikryl@nrcan.gc.ca

'Geomagnetic Laboratory, Natural Resources Canada, 2617 Anderson Rd, Ottawa, ON K1A 0E7, Canada

Full list of author information is available at the end of the article
}

scintillation with the phase scintillation primarily caused by steep ionospheric density gradients and irregularities associated with auroral and cusp precipitation and polar cap patches (Spogli et al. 2009; Li et al. 2010; Prikryl et al. 2011a, b, 2013a; Jiao et al. 2013). The ionospheric dynamics in these regions is driven by the coupling between solar wind, the magnetosphere, and ionosphere. Solar wind disturbances, in particular, the co-rotating interaction regions (CIRs) on the leading edge of highspeed streams (HSSs) and interplanetary coronal mass ejections (ICMEs) have been closely linked with the occurrence of scintillation at high latitudes (Prikryl et al. 2012). These initial results demonstrated a technique of probabilistic forecast of phase scintillation occurrence in the cusp relative to arrival times of CIRs and ICMEs. Such scintillation prediction can be combined with assessment of the GNSS receiver tracking performance 
(Prikryl et al. 2013b) and thus offer a potentially useful tool to provide users with expected tracking conditions. In this paper, we extended the superposed epoch (SPE) analysis of solar wind parameters and scintillation occurrence over a period of 5 years (2008 to 2012) and obtained probability distribution functions that can be used in probabilistic forecasting of phase scintillation and cycle slip occurrence at high latitudes.

\section{Methods}

Phase scintillation data used in this study were collected from 2008 and 2012 by ten specialized GPS ionospheric scintillation and total electron content (TEC) monitors (GISTMs) of the Canadian High Arctic Ionospheric Network (CHAIN) (http://chain.physics.unb.ca/chain) (Jayachandran et al. 2009). CHAIN instruments, including six ionosondes, are distributed in the auroral oval, cusp, and the polar cap (Figure 1). A brief description of CHAIN, the scintillation parameters obtained, and the geographic and corrected geomagnetic coordinates of stations can be found in Prikryl et al. (2011a).

The NovAtel OEM4 GSV 4004B dual frequency GPS receivers (Van Dierendonck and Arbesser-Rastburg 2004), with special firmware specifically configured to record the power and phase of the $\mathrm{L} 1$ signal at $50-\mathrm{Hz}$ sampling rate, compute the ionospheric TEC using both L1 and L2 signals, the amplitude scintillation index $S_{4}$, and the phase scintillation index $\sigma_{\Phi}$. The phase scintillation index $\sigma_{\Phi}$ is the standard deviation of the detrended phase using a filter in the receiver with $0.1-\mathrm{Hz}$ lower cutoff. Intense scintillation is usually accompanied by cycle slips. A cycle slip

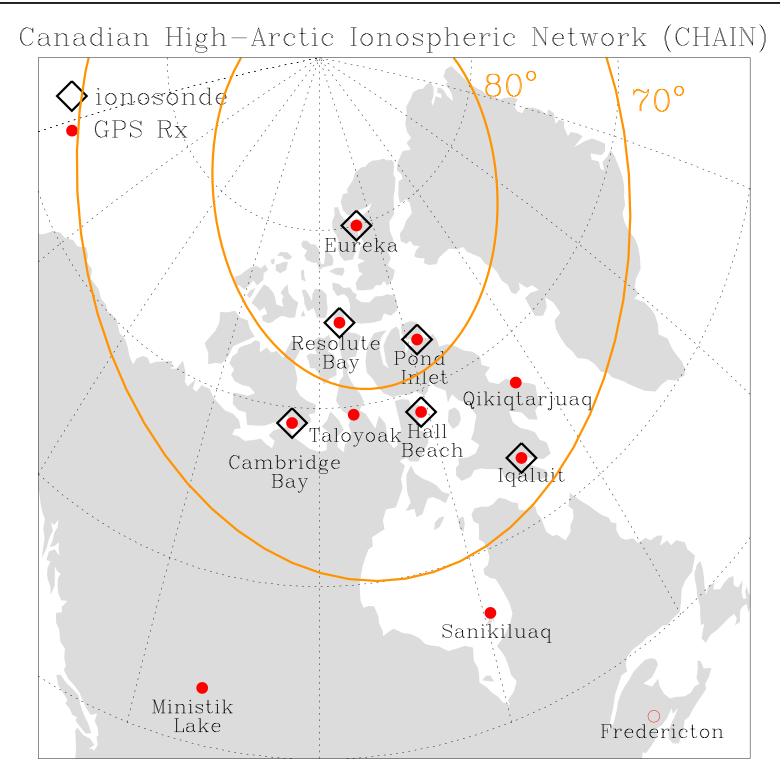

Figure 1 Canadian High Arctic lonospheric Network (CHAIN). The corrected geomagnetic (CGM) latitudes $70^{\circ}$ and $80^{\circ}$ are superposed over the geographic grid.
(Horvath and Crozier 2007) is defined here as a jump in differential phase TEC of more than or equal to $1.5 \mathrm{TECU}$ in $1 \mathrm{~s}$ ( 1 TEC unit corresponds to $10^{16}$ electrons $\left./ \mathrm{m}^{2}\right)$.

Solar wind data, projected to the Earth's bow shock, are obtained from the Goddard Space Flight Center Space Physics Data Facility OMNIWeb (http://omniweb. gsfc.nasa.gov/). To characterize the solar wind by one index, we use the quasi-invariant (QI) index (Osherovich et al. 1999) that is defined as the ratio of the solar-wind magnetic to ram pressures.

High-speed streams generate CIRs, regions between the fast and slow solar wind where the solar wind plasma is compressed. Following the criteria of (Prikryl et al. 2012), arrival times of high-speed stream interfaces CIR/HSSs are determined. A total of $108 \mathrm{HSS} / \mathrm{CIRs}$ with a maximum velocity $V_{\text {MAX }}$ exceeding $500 \mathrm{~km} / \mathrm{s}$ are used in the SPE analysis of scintillation data from 2008 to 2012. For ICMEs, we use a catalogue of near-Earth ICMEs (Richardson and Cane 2010) that is updated at http://www.srl.caltech.edu/ACE/ASC/DATA/level3. In this table, ICMEs are classified into three categories based on the properties of magnetic clouds in column $l$ : a value of 2 in this column indicating that a magnetic cloud (MC) has been reported in association with the ICME; a value of 1 indicating that the ICME shows evidence of a rotation in field direction but lacks some other characteristics of an MC, for example, an enhanced magnetic field; and a value of 0 indicating that the ICME has not been a reported magnetic cloud and lacks most of the typical features of a magnetic cloud, such as a smoothly rotating, enhanced magnetic field.

The time of the leading edge (front) of an HSS or of the ICME upstream shock/wave/disturbance provides key times of a geoeffective solar wind disturbance observed upstream from the Earth. Projected to the Earth's bow shock (OMNI), these key arrival times can be used in an SPE analysis to obtain an average response of the ionosphere, e.g., geomagnetic disturbance (McPherron and Siscoe 2004; McPherron and Weygand 2006), riometer absorption (Kavanagh et al. 2012), and scintillation and cycle slip occurrence (Prikryl et al. 2012). Such results can then be used in probabilistic forecasting of a given ionospheric variable due to specific solar wind conditions. This can be thought of as an analogy to traditional meteorology approach in weather forecasting based on atmospheric air mass climatologies separated by boundaries (weather fronts) as discussed by McPherron and Siscoe (2004).

\section{SPE analysis of solar wind and scintillation data}

To obtain the mean variations of solar wind parameters relative to HSS interface (CIR) SPE analysis of solar wind plasma time series is performed. Figure 2a shows the mean solar wind velocity, $V$, mean flow angle $\Phi_{V}$, density, 


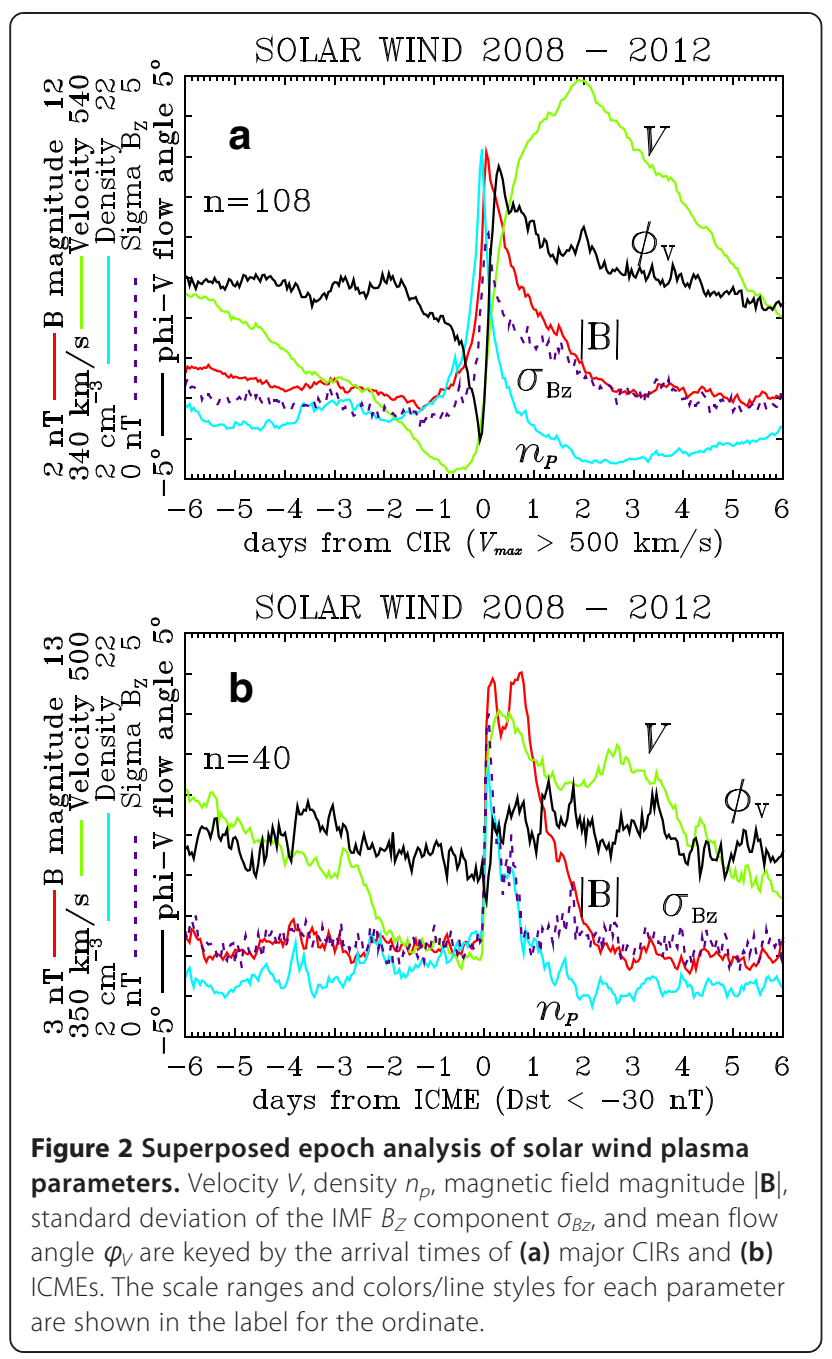

$n_{p}$, magnetic field magnitude, $|\mathbf{B}|$, and standard deviation of IMF $B_{Z}$ component, $\sigma_{B z}$, over 12 days centered at the key time (day 0 ). The mean solar wind velocity decreases to a minimum just before the stream interface and then steeply rises to a maximum 2 days later. All other mean solar wind parameters peak at, or very close to, the key time. The mean flow angle $\Phi_{V}$ shows a bipolar east-west deflection. Figure $2 \mathrm{~b}$ shows the results of the SPE analysis for 40 ICMEs for which the geomagnetic disturbance, Dst, was less than $-30 \mathrm{nT}$. Because the ICMEs are often preceded by shocks, the mean $V$, $n_{p},|\mathbf{B}|$, and $\sigma_{B z}$ increase near the key time. The mean flow angle $\Phi_{V}$ does not show a strong bipolar east-west deflection that is typical for CIRs. These results are similar to those obtained previously (Prikryl et al. 2012) for 2008 to 2010.

The GPS phase scintillation predominantly occurs in the ionospheric footprint of the cusp, auroral oval, and polar cap (Spogli et al. 2009; Prikryl et al. 2011a, b, 2012). In response to varying geomagnetic activity, the scintillation regions shift in latitude. To examine a response of scintillation and to propose a method of scintillation forecasting relative to arrival time of CIRs and ICMEs, Prikryl et al. (2012) focused on the cusp stations in Cambridge Bay and Taloyoak that showed steep increases in scintillation occurrence on the zero epoch day tapering off a few days later. We now include ten CHAIN stations in the SPE analysis. The scintillation and cycle slip occurrence is mapped as a function of CGM latitude and magnetic local time (MLT).

Similarly to solar wind parameters in Figure 2, Figure 3a,b shows the SPE analysis results for QI and $K p$ indices, shown in green and black lines, respectively. The mean QI index increases more gradually and peaks later but reaches a higher value for ICMEs than for HSS/CIRs. This is because many ICMEs are actually magnetic clouds, and the ratio of the magnetic to ram pressures is higher inside the magnetic cloud. Also, the average background levels of QI

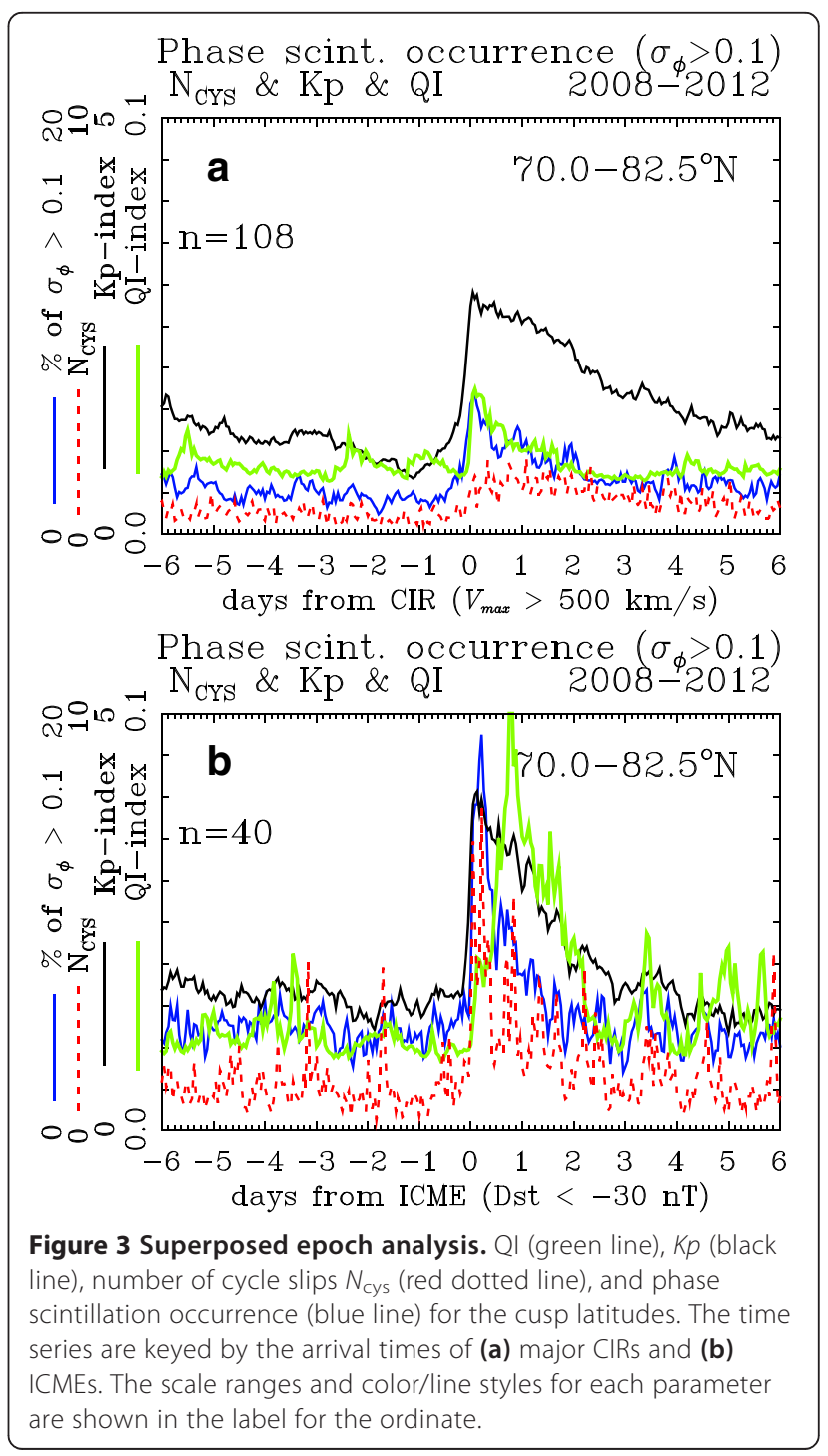


and $K p$ before the arrival of a solar wind disturbance are higher for ICMEs than for HSS/CIRs because most of the ICMEs occurred in the years of rising solar activity (2010 to 2012), while the HSS/CIRs events are distributed more evenly over the whole period. However, the focus of this study is phase scintillation occurrence and number of cycle slips $N_{\text {cys }}$ that are shown in Figure 3a,b in blue and red lines, respectively. They are averaged over the cusp region defined here by latitude range from $70.0^{\circ} \mathrm{N}$ to $82.5^{\circ} \mathrm{N}$ and MLT from 0600 to 1800 hours. Both the scintillation and cycle slip occurrence peaked about three to four times higher for ICMEs than for HSS/CIRs. As we already noted for QI and $K p$ indices, the background average levels of occurrences are higher for the case of ICMEs than for HSS/CIRs.

Figures 4 and 5 show the SPE analysis results for CIRs and ICMEs, respectively, for all magnetic latitudes and MLTs. Figure 4a,b shows the SPE analysis results based on 108 CIRs, namely, the maps of percentage occurrence of phase scintillation $\sigma_{\Phi}$ exceeding 0.1 radians and average number of cycle slips, respectively. The positions of the statistical auroral oval (Feldstein and Starkov 1967; Holzworth and Meng 1975) are superposed in white line for conditions from very quiet (IQ $=0$ ) to disturbed $(\mathrm{IQ}=6)$, proportionally to the daily mean value of the $K p$ index. For each epoch day, the phase scintillation occurrence and mean number of cycle slips maximized in the cusp and extended into the polar cap with the grand maximum occurring on the epoch day 0 . However, significant scintillation also occurred at auroral and even at subauroral latitudes. The occurrence of scintillation and cycle slips tapered off, reaching the pre-event background level a few days later.

Figure 5a,b shows the results of the SPE analysis based on 40 ICMEs (Dst $<-30 \mathrm{nT}$ ). Similarly to CIRs, the mean phase scintillation occurrence and mean number of cycle slips maximized in the cusp and extended into the polar cap with the grand maximum occurring on the epoch day 0 , but the peak scintillation occurrence and cycle slip number were higher and tapered off faster, reaching the pre-event background level on epoch day 2 . Significant scintillation also occurred at auroral and even subauroral latitudes on epoch days 0 and 1 .

\section{Cumulative probability distribution functions}

Figures 6 and 7 show cumulative probability distribution functions (PDFs) for the phase scintillation occurrence in the 'cusp' region defined here by latitude range from $70.0^{\circ} \mathrm{N}$ to $82.5^{\circ} \mathrm{N}$ and MLT from 0600 to 1800 hours. The PDFs are based on the SPE analysis results shown in Figures $4 \mathrm{a}$ and $5 \mathrm{a}$ for CIRs and ICMEs, respectively. The curves represent the probabilities that phase scintillation will exceed a given value as plotted on the abscissa.

In Figure 6, the solid lines show overall PDFs for epoch days -1 and 0 (for slow solar wind just before and fast solar wind after the key time) including all solar wind conditions. The overall probability curves for both the slow and fast streams (shown by solid lines) are bracketed by probability curves (broken lines) for scintillation data subdivided by corresponding solar wind low and high (below and above median) values of $V,|\mathbf{B}|$, and $\sigma_{B z}$. In general, the probabilities are higher for ICMEs
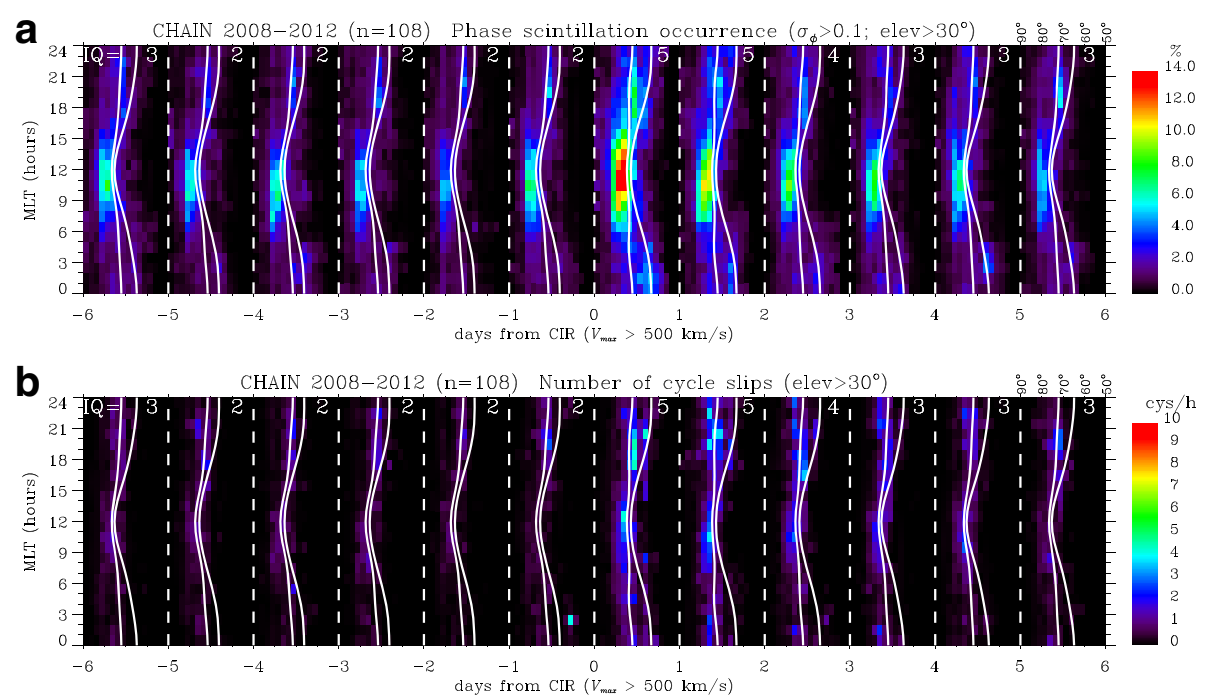

Figure 4 The SPE analysis results based on $108 \mathrm{CIRs}$. The maps of (a) an average percentage occurrence of phase scintillation $\sigma_{\Phi}$ exceeding 0.1 radians and (b) average number of cycle slips for epoch days before and after the arrival of CIRs. The positions of the statistical auroral oval are superposed in solid white lines for conditions from very quiet $(I Q=0)$ to very disturbed $(I Q=6)$, proportionally to the daily mean value of the $K p$ index. 

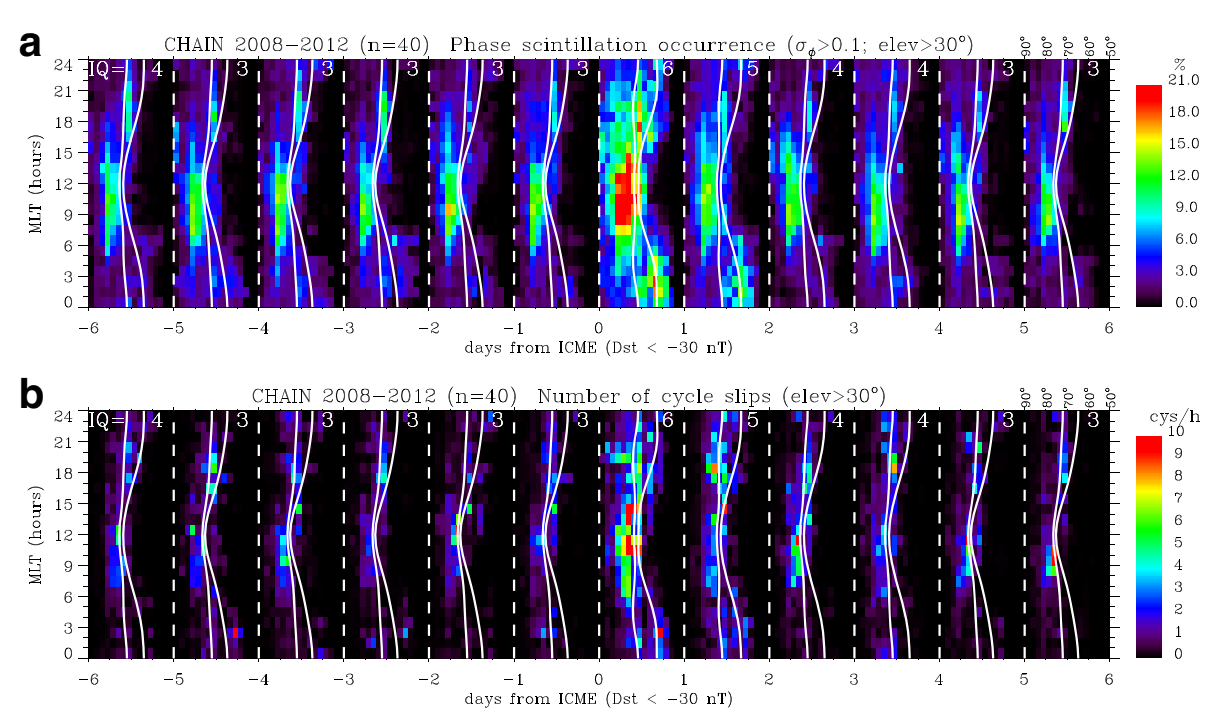

Figure 5 Same as Figure $4 a, b$ but for the SPE analysis based on 40 ICMEs.

than for CIRs. As discussed further in the 'Results and discussion' section, the period of slow solar wind before the arrival of CIR/HSS is characterized by very low geomagnetic activity and, expectedly, the lowest level of scintillation. It gives the lowest background levels of scintillation occurrence and thus the lowest probabilities, particularly for the case of low (below median) values of $|\mathbf{B}|$ and $\sigma_{B z}$. Because ICMEs can arrive when a HSS is active, the mean 'background' level of probabilities before the arrival of ICMEs is expectedly higher. By the same token, the scintillation occurrence (probability) level after an ICME arrival is a superposition of that 'background' level and scintillation caused by the ICME interaction with the magnetosphereionosphere system. The PDFs shown for low and high $V$, $|\mathbf{B}|$, and $\sigma_{B z}$ indicate that variability about the average of the solar wind speed affects the computed probability values the least. The scintillation occurrence (probability) is strongly affected by the variability of the magnetic field.
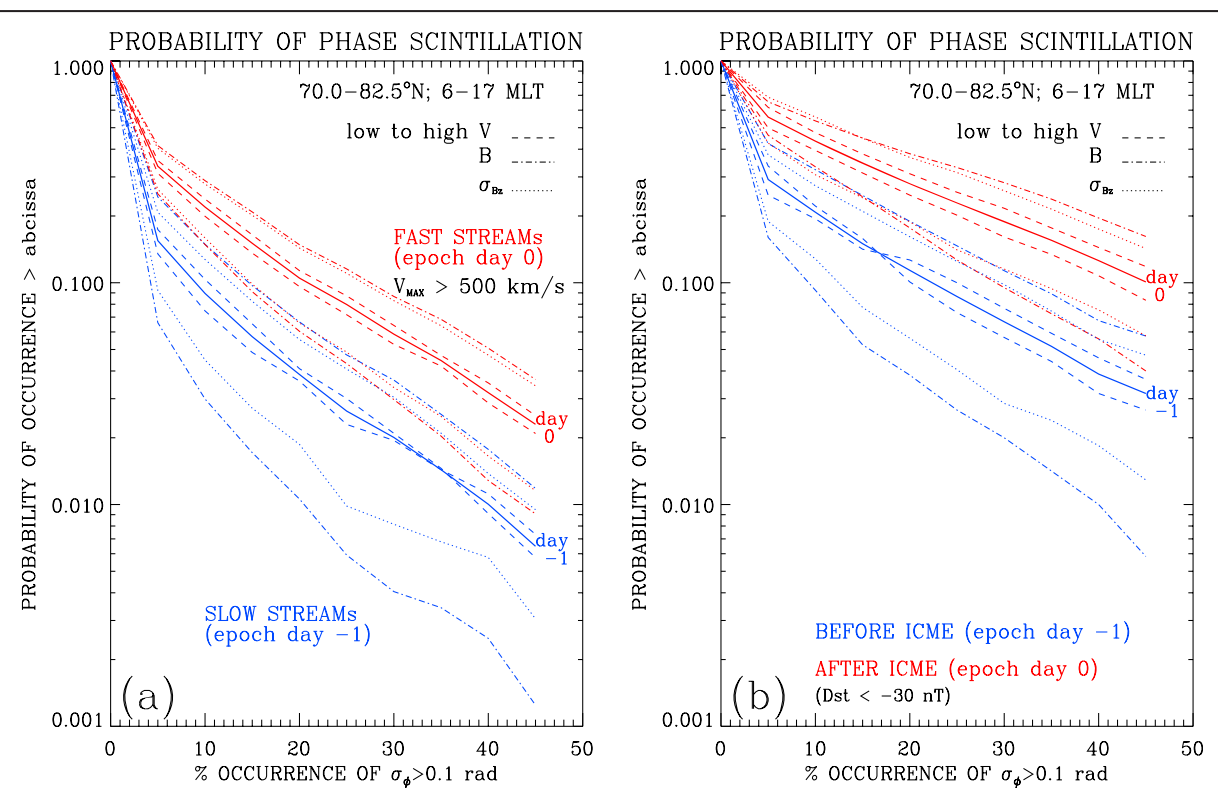

Figure 6 The cumulative PDFs in the 'cusp' for days -1 and 0 . Probability of the phase scintillation occurrence for (a) slow and fast solar wind on days just before and after CIRs $(n=108)$ and $(\mathbf{b})$ days just before and after ICMEs (Dst $<-30 \mathrm{nT} ; n=40)$. The overall probabilities for each case (solid lines) are bracketed by a pair of curves (broken lines) for data corresponding to low and high (below and above median) values of the mean solar wind velocity, $V$ (dashed lines), magnetic field magnitude, $|\mathbf{B}|$ (dash-dot line), and standard deviation of IMF $B_{Z}$ component, $\sigma_{B Z}$ (dotted line). 

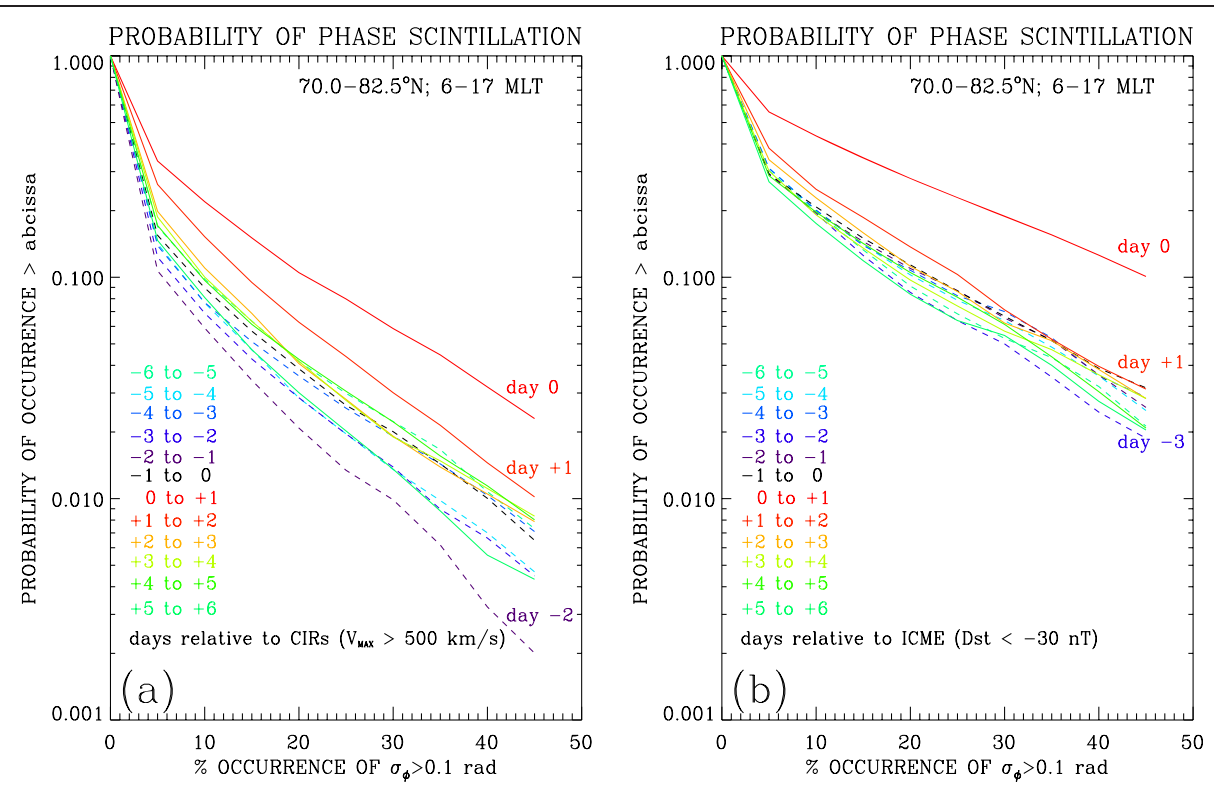

Figure 7 The cumulative PDFs in the 'cusp' for days -6 to +6 . Probability of the phase scintillation occurrence stepped by 1 -day intervals before and after (a) CIRs ( $n=108$ ) and (b) ICMEs (Dst $<-30 \mathrm{nT} ; n=40$ ). The line colors correspond to days before and after CIR/ICME as shown in the legend. The days before CIR/ICME are shown in broken lines (from black to blue to cyan); the days after CIR/ICME are shown in solid lines (red to yellow to green).

In Figure 7a,b, the overall PDFs are shown from -6 to +6 epoch days stepped by 1-day intervals for HSS/CIRs and ICMEs, respectively. In both cases, the highest probabilities are observed for day 0 (i.e., over $24 \mathrm{~h}$ after the key time) but are significantly higher for ICMEs. Although the background levels of occurrences, and thus the PDF values, are higher for the case of ICMEs than for HSS/CIRs; the range of probabilities is larger for the latter. It is noted that the lowest occurrences (Figures 3a and 4) and the lowest probabilities (Figures $7 \mathrm{a}$ and $8 \mathrm{~b}$ ) are observed 2 days before HSS/CIR arrivals, i.e., on epoch day -2 .

To differentiate between the response in the cusp and auroral zone, Figures 8 and 9 show the cumulative PDFs for the phase scintillation occurrence in the nighttime auroral zone defined here by a latitude range from $60.0^{\circ} \mathrm{N}$ to $75.0^{\circ} \mathrm{N}$ and MLT from 1800 to 0600 hours. Clearly, the
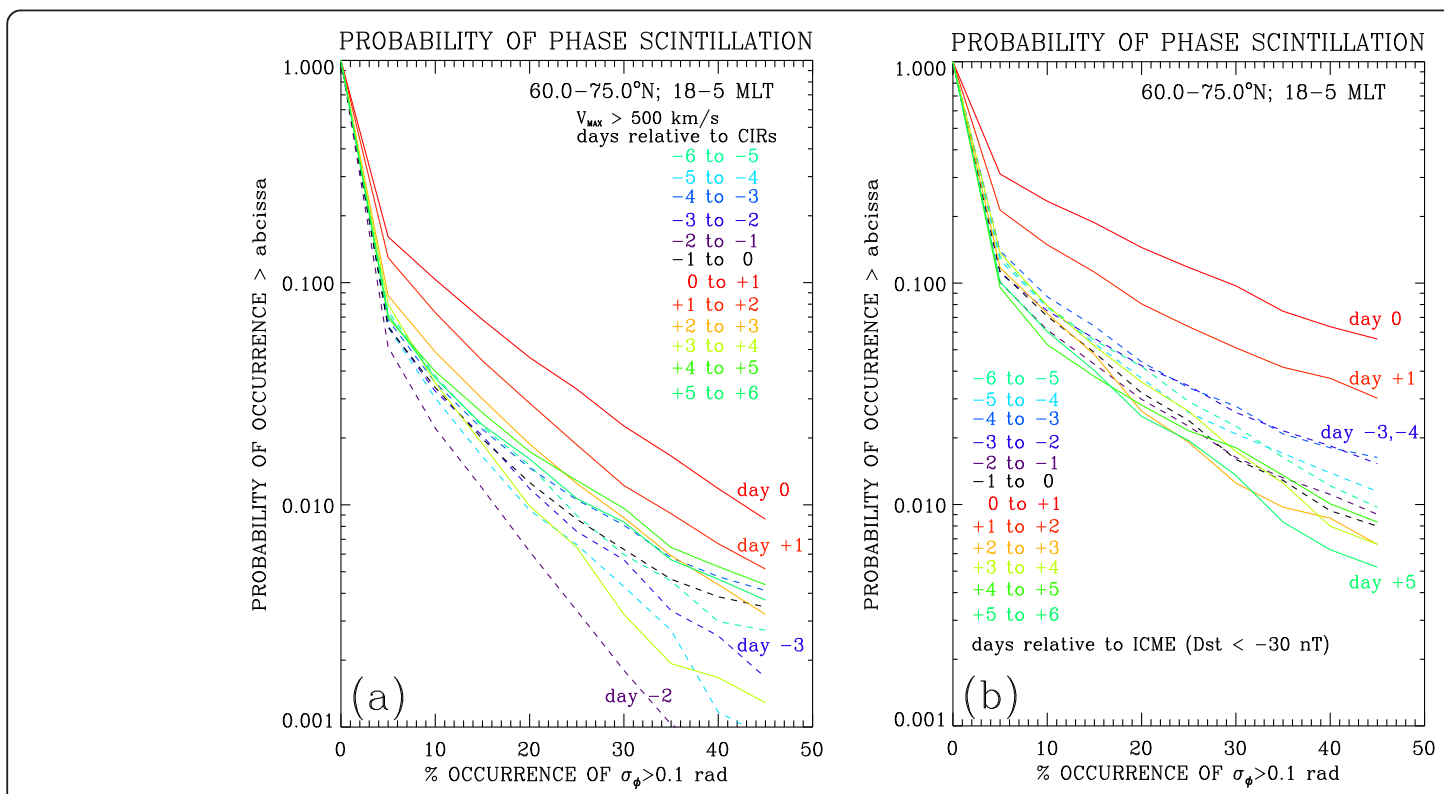

Figure 8 Same as Figure 7a,b but showing the cumulative PDFs for the nightside auroral zone. 

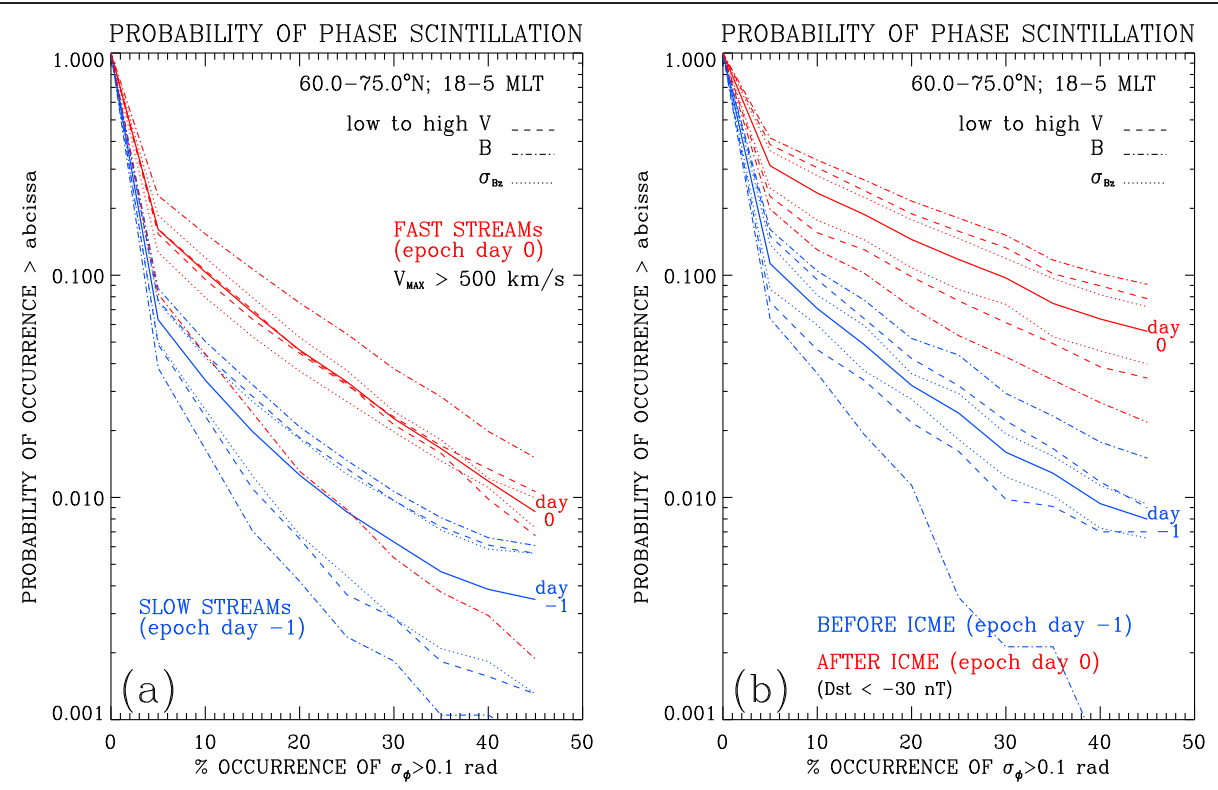

Figure 9 Same as Figure $6 a, b$ but showing the cumulative PDFs for the nightside auroral zone.

probabilities are significantly lower as already indicated by lower occurrence rate of phase scintillation in the auroral oval as compared with the cusp (Figures 4a and 5a). However, in all instances, both in the cusp and in the auroral zone, the highest probability of phase scintillation occurrence is on epoch day 0, i.e., just after the arrival of CIRs or ICMEs. Also, the probabilities are higher for larger values of $|\mathbf{B}|, \sigma_{B z}$, and $V$. The variability of the magnetic field affects the probabilities the most.

\section{Results and discussion}

Because of complexity of the solar wind-magnetosphereionosphere coupling and the resulting ionospheric irregularities, it is generally very difficult or impossible to predict radio scintillation for a given ray path based on physical modeling of the ionospheric structure. Thus, probabilistic forecasting based on statistical results over many years for specific conditions either in solar wind or in the ionosphere appears to be appropriate approach in forecasting scintillation at high latitudes. Since the solar wind disturbances like CIRs and ICMEs are the major causes of geomagnetic and ionospheric disturbances that result in enhanced scintillation, we applied the forecasting method by McPherron and Siscoe (2004) to forecast phase scintillation occurrence at high latitudes. Initial results (Prikryl et al. 2012) have demonstrated the feasibility of probabilistic forecasting of scintillation in the cusp for a given location of a GPS receiver. In this paper, we use the statistical data from a network of receivers to map the scintillation occurrence as a function of magnetic latitude and MLT. Focusing on specific solar wind events (CIRs and ICMEs), the SPE analysis can provide average occurrence rate and average number of cycle slips relative to arrival time of CIRs or ICMEs, as a function of magnetic latitude and MLT. Based on these statistical results, one can then construct cumulative PDFs. The PDFs can be specified for a range of magnetic latitudes and MLTs, and also for different solar wind conditions. They can be then used in probabilistic forecasting of scintillation occurrence at high latitudes.

GPS scintillation databases with improved spatial coverage in latitude/longitude including polar cap and auroral oval, as well as coverage over a complete solar cycle, are needed. The present data cover mostly the last, anomalously extended, solar minimum and rise of solar cycle 24, and the majority of ICMEs caused only weak geomagnetic disturbances; none of the ICMEs resulted in a major geomagnetic storm, e.g., Richardson (2013), which is clearly not representative of periods of high solar activity. In comparison, strong and recurrent high-speed streams from coronal holes remained active during most of the period studied in this paper. The obtained mean probability curves for days before and after CIR arrival are expected to be similar throughout the solar cycle, although they may reflect the differences between slow and fast solar winds that are likely to be enhanced during the declining phase when the recurrent streams are dominant.

As we have already discussed in the 'Cumulative probability distribution functions' section, the SPE analysis keyed by the HSS/CIR arrivals resulted in lower background levels of occurrences and probabilities (before epoch day 0 ), which is partly because of large contribution from years of solar minimum when HSSs regularly occur, while ICMEs are mostly absent. The lowest 
scintillation occurrences and lowest probabilities are found for epoch day -2 , i.e., 2 days before HSS/CIR arrival. This is consistent with the periods of 'calm' (Borovsky and Steinberg 2006) during 2 days of slow solar wind just before the major CIR/HSS arrival that is followed by about two 'stormy' days of fast solar wind disturbances. The latter solar wind variation is then mirrored by geomagnetic activity (Figure 3a) as well as scintillation and cycle slip occurrence.

MCs are a subset of ICMEs with a smoothly rotating and stronger-than-average magnetic field, as well as low proton temperature (Burlaga et al. 1981). ICMEs associated with MCs are known to be more geoeffective (Huttunen et al.
2005) than non-MC ICMEs. Even small-scale magnetic ropes are found to induce substorm activity (Zhang et al. 2013). Strong ionospheric scintillation was observed during the magnetic cloud-induced geomagnetic storm of April 5 to 7, 2010 (Prikryl et al. 2011b). The scintillation occurrence during that event increased in the auroral oval in both the northern and southern hemispheres as the MC magnetic field rotated southward. As already discussed in the 'Methods' section, we use the MC classification of ICMEs based on the catalogue of ICMEs by Richardson and Cane (2010). Out of 40 ICMEs (Dst $<-30 \mathrm{nT}$ ) used in the present SPE analysis (Figure 5), 27 cases were classified as MCs indicating all required characteristics of MCs
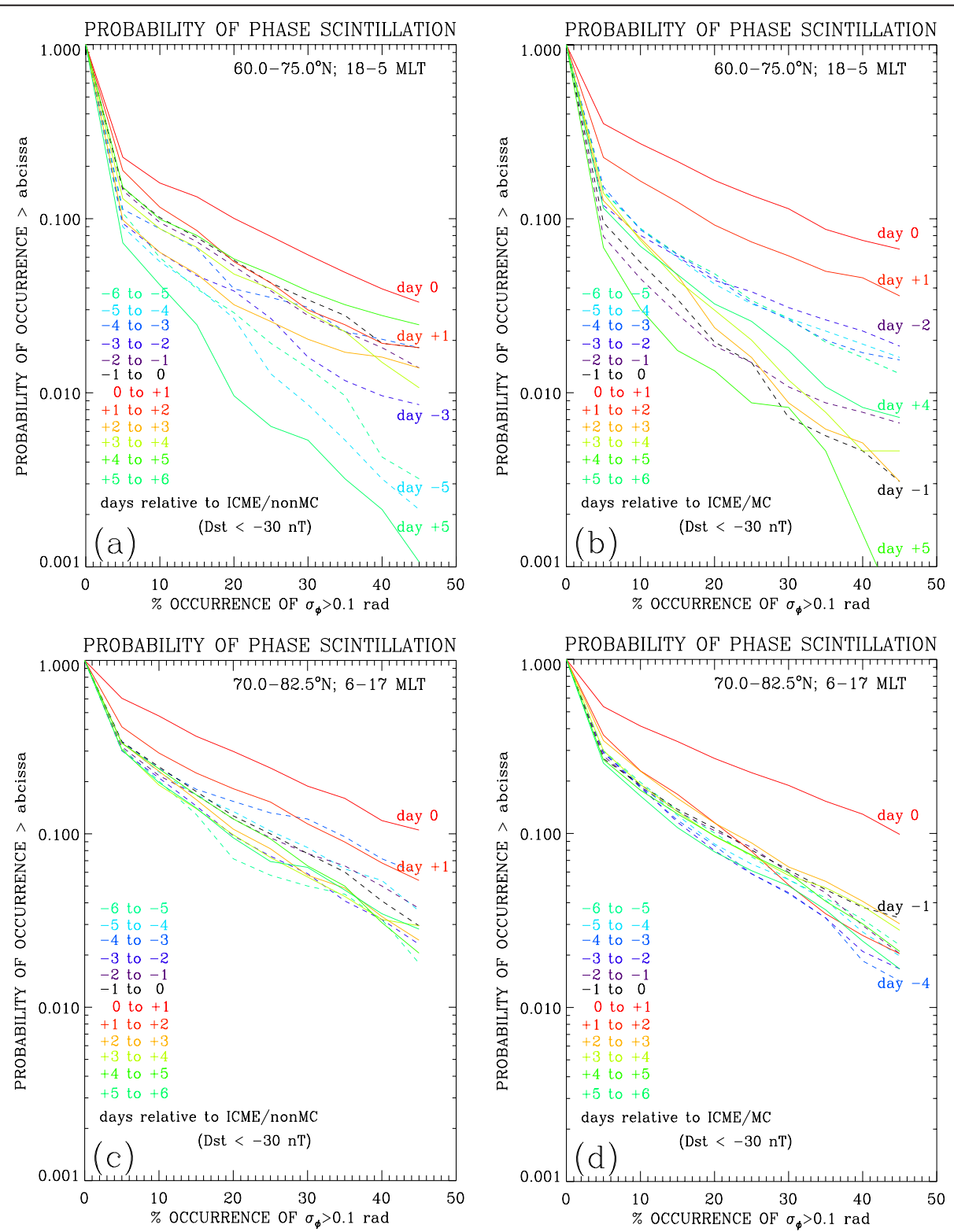

Figure 10 The cumulative PDFs for MC and non-MC ICMEs. Probability of the phase scintillation occurrence in the 'nightside auroral zone' (top panels) and in the 'cusp' (bottom panels) for $(\mathbf{a}, \mathbf{c})$ non-MC $(n=13)$ and $(\mathbf{b}, \mathbf{d}) \mathrm{MC}(n=27)$ events. 
$(l=2)$, while 13 cases lacked some or all important characteristics of MCs $(l=0$ or 1$)$.

Figure 10a,b,c,d shows the PDFs for 'nightside auroral zone' (Figure 10a,b) and 'cusp' (Figure 10c,d) obtained by the SPE analysis for the subsets of MC $(n=27)$ and non-MC $(n=13)$ events. In the 'nightside auroral zone', the probabilities of scintillation occurrence just after arrival are significantly higher for $\mathrm{MC}$ events (Figure 10b) compared to the non-MC events (Figure 10a). This is consistent with the finding that $\mathrm{MCs}$ are associated with increased substorm activity (Huttunen et al. 2005; Zhang et al. 2013). In the 'cusp', while the PDFs just after the arrival of an event (epoch day 0) are very similar for non-MC (Figure 10c) and MC (Figure 10d) events, the 'background' PDFs before and a few days after the epoch day 0 are lower for the MC subset (Figure 10d). This suggests that, both in the cusp and auroral oval, the difference between the probability of scintillation occurrence just after ICME arrival and probability of scintillation occurrence before and a few days after the event is higher for the $\mathrm{MC}$ events than for the non-MC events.

These results are based on a relatively small statistical sample, and while they represent a proof of concept, a larger statistical sample is needed to further refine the scintillation forecasting method by compiling PDFs separately for different seasons, levels of solar activity, various threshold values of $\sigma_{\Phi}$, and different magnetic latitudes and local times. Also, it will be important to consider periods of southward and northward IMF since the IMF $B_{Z}$ component (in the geocentric solar magnetospheric coordinate system (GSM)) controls the coupling to the magnetosphere and the energy deposition into the ionosphere, which, in turn, affects the ionospheric structure and thus the scintillation level.

As already discussed previously (Prikryl et al. 2012), the feasibility of probabilistic forecasting of scintillation relative to solar wind disturbances will be dependent on accurate prediction of CIR or CME arrivals. Space weather forecasting has benefited from advances in MHD modeling of CMEs and CIRs (Odstrcil et al. 2005; Wu et al. 2011). Multi-spacecraft tracking of CIRs propagation (Williams et al. 2011; Davis et al. 2012) and forecasting propagation and evolution of CMEs in an operational setting (Zheng et al. 2013) are being developed. The viability of probabilistic forecasting of ionospheric scintillation will hinge upon the progress of the solar wind modeling and monitoring efforts to improve space weather forecasting in general.

\section{Conclusions}

The SPE analysis is applied to time series of phase scintillation and cycle slip occurrence observed by CHAIN from 2008 to 2012. Arrival times of HSS and ICME were used as key times for the SPE analysis. Mapped as a function of magnetic latitude and magnetic local time, phase scintillation and cycle slips occur predominantly on the dayside in the cusp and in the nightside auroral oval. The scintillation and cycle slips peak on days when HSS or ICME impacts the Earth's magnetosphere. The occurrence of phase scintillation and cycle slips taper off more gradually and reach the mean background levels a few days after the arrival of HSS. In contrast, the occurrence peaks significantly higher just after the ICME impact but drops to background levels 1 or 2 days after the ICME arrivals. The ICMEs of magnetic cloud type result in higher occurrence of scintillation and cycle slips in the nightside auroral oval when compared to the nonmagnetic cloud ICMEs. Cumulative probability distribution functions for the phase scintillation occurrence as a function of epoch day relative to the HSS or ICME arrival are obtained for typical cusp and auroral oval locations. The probability distribution functions can also be specified for low and high (below and above median) values of various solar wind plasma parameters. These results can be used to predict occurrence of phase scintillation and cycle slip occurrence; however, a larger statistical dataset covering at least one solar cycle is needed to develop a probabilistic forecasting method.

\section{Competing interests}

The authors declare that they have no competing interests.

\section{Authors' contributions}

PP designed this study, analyzed the data, and wrote the manuscript. PTJ conceived of and implemented the CHAIN project and helped in interpretation of the data. SCM contributed to the analysis and interpretation of the GPS data. IGR maintained the archive of the ICMEs and helped in the interpretation of the solar wind data. All authors read and approved the final manuscript.

\section{Acknowledgements}

Infrastructure funding for CHAIN was provided by the Canada Foundation for Innovation and the New Brunswick Innovation Foundation. CHAIN operation is conducted in collaboration with the Canadian Space Agency (CSA). The solar wind data were obtained from Goddard Space Flight Center Space Physics Data Facility OMNIWeb (http://omniweb.gsfc.nasa.gov/). This work was supported by the Public Safety Geosciences program of the Natural Resources Canada, Earth Sciences Sector (NRCan ESS Contribution Number 20130496).

\section{Author details}

${ }^{1}$ Geomagnetic Laboratory, Natural Resources Canada, 2617 Anderson Rd, Ottawa, ON K1A 0E7, Canada. ${ }^{2}$ Physics Department, University of New Brunswick, Fredericton, NB E3B 5A3, Canada. ${ }^{3}$ Department of Physics and Astronomy, University of Calgary, Calgary, AB T2N 1N4, Canada. ${ }^{4}$ CRESST and Department of Astronomy, University of Maryland, College Park, MD 20742, USA. ${ }^{5}$ NASA Goddard Space Flight Center, Greenbelt, MD 20771, USA.

Received: 12 March 2014 Accepted: 12 May 2014

Published: 30 June 2014

\section{References}

Aarons J (1982) Global morphology of ionospheric scintillations. Proc IEE 70 (4):360-378

Aarons J (1997) Global positioning system phase fluctuations at auroral latitudes. J Geophys Res 102(A8):17219-17231, doi:10.1029/97JA01118

Aarons J, Lin B, Mendillo M, Liou K, Codrescu M (2000) Global positioning system phase fluctuations and ultraviolet images from the polar satellite. J Geophys Res 105(A3):5201-5213, doi:10.1029/1999JA900409 
Basu S, MacKenzie EM, Basu S, Costa E, Fougere PF, Carlson HC Jr, Whitney HE (1987) $250 \mathrm{MHz} / \mathrm{GHz}$ scintillation parameters in the equatorial, polar, and auroral environments. IEEE J Select Areas Commun SAC-2(2):102-115

Basu S, Basu S, Sojka JJ, Schunk RW, MacKenzie E (1995) Macroscale modeling and mesoscale observations of plasma density structures in the polar cap. Geophys Res Lett 22(8):881-884, doi:10.1029/95GL00467

Basu S, Weber EJ, Bullett TW, Keskinen MJ, Mackenzie E, Doherty P, Sheehan R, Kuenzler H, Ning P, Bongiolatti J (1998) Characteristics of plasma structuring in the cusp/cleft region at Svalbard. Radio Sci 33(6):1885-1899, doi:10.1029/ 98RS01597

Borovsky JE, Steinberg JT (2006) The "calm before the storm" in CIR/ magnetosphere interactions: occurrence statistics, solar wind statistics, and magnetospheric preconditioning. J Geophys Res 111:A07S10, doi:10.1029/ 2005JA011397

Burlaga L, Sittler E, Mariani F, Schwenn R (1981) Magnetic loop behind an interplanetary shock - Voyager, Helios, and IMP 8 observations. J Geophys Res 86:6673-6684

Conker RS, El Arini MB, Hegarty CJ, Hsiao T (2003) Modeling the effects of ionospheric scintillation on GPS/satellite-based augmentation system availability. Radio Sci 38(1):1001, doi:10.1029/2000RS002604

Davis CJ, Davies JA, Owens MJ, Lockwood M (2012) Predicting the arrival of high-speed solar wind streams at Earth using the STEREO Heliospheric Imagers. Space Weather 10:S02003, doi:10.1029/2011SW000737

Feldstein Yl, Starkov GV (1967) Dynamics of auroral belt and polar geomagnetic disturbances. Planet Space Sci 15:209-230

Holzworth RH, Meng C-I (1975) Mathematical representation of the auroral oval. Geophys Res Lett 2(9):377-380

Horvath I, Crozier S (2007) Software developed for obtaining GPS-derived total electron content values. Radio Sci 42:RS2002, doi:10.1029/2006RS003452

Huttunen KEJ, Schwenn R, Bothmer V, Koskinen HEJ (2005) Properties and geoeffectiveness of magnetic clouds in the rising, maximum and early declining phases of solar cycle 23. Ann Geophys 23:625-641, doi:10.5194/ angeo-23-625-2005

Jayachandran PT, Langley RB, MacDougall JW, Mushini SC, Pokhotelov D, Hamza AM, Mann IR, Milling DK, Kale ZC, Chadwick R, Kelly T, Danskin DW, Carrano CS (2009) Canadian High Arctic lonospheric Network (CHAIN). Radio Sci 44: RSOA03, doi:10.1029/2008RS004046 (printed 45(1), 2010)

Jiao Y, Morton YT, Taylor S, Pelgrum W (2013) Characterization of high-latitude ionospheric scintillation of GPS signals. Radio Sci 48:698-708, doi:10.1002/ 2013RS005259

Li G, Ning B, Ren Z, Hu L (2010) Statistics of GPS ionospheric scintillation and irregularities over polar regions at solar minimum. GPS Solut 14:331-341, doi:10.1007/s10291-009-0156-X

Kavanagh AJ, Honary F, Donovan EF, Ulich T, Denton MH (2012) Key features of $>30$ keV electron precipitation during high speed solar wind streams: a superposed epoch analysis. J Geophys Res 117:A00L09, doi:10.1029/ 2011JA017320

McPherron RL, Siscoe G (2004) Probabilistic forecasting of geomagnetic indices using solar wind air mass analysis. Space Weather 2:S01001, doi:10.1029/ 2003SW000003

McPherron RL, Weygand J (2006) The solar wind and geomagnetic activity as a function of time relative to corotating interaction regions. In: Tsurutani $B$, McPherron RL, Gonzalez W, Lu G, Sobral JHA, Gopalswamy N (eds) Recurrent magnetic storms: corotating solar wind streams. AGU Monograph. AGU, Washington, D.C, pp 125-137, 167

Odstrcil D, Pizzo VJ, Arge CN (2005) Propagation of the 12 May 1997 interplanetary coronal mass ejection in evolving solar wind structures. J Geophys Res 110:A02106, doi;10.1029/2004JA010745

Osherovich VA, Fainberg J, Stone RG (1999) Solar wind quasi-invariant as a new index of solar activity. Geophys Res Lett 26(16):2597-2600

Prikryl P, Jayachandran PT, Mushini SC, Chadwick R (2011a) Climatology of GPS phase scintillation and HF radar backscatter for the high-latitude ionosphere under solar minimum conditions. Ann Geophys 29:377-392, doi:10.5194/ angeo-29-377-2011

Prikryl P, Spogli L, Jayachandran PT, Kinrade J, Mitchell CN, Ning B, Li G, Cilliers PJ, Terkildsen M, Danskin DW, Spanswick E, Donovan E, Weatherwax AT, Bristow WA, Alfonsi L, De Franceschi G, Romano V, Ngwira CM, Opperman BDL (2011b) Interhemispheric comparison of GPS phase scintillation at high latitudes during the magnetic-cloud-induced geomagnetic storm of 5-7 April 2010. Ann Geophys 29:2287-2304, doi:10.5194/angeo-29-2287-2011
Prikryl P, Jayachandran PT, Mushini SC, Richardson IG (2012) Toward the probabilistic forecasting of high-latitude GPS phase scintillation. Space Weather 10:S08005, doi:10.1029/2012SW000800

Prikryl P, Ghoddousi-Fard R, Kunduri BSR, Thomas EG, Coster AJ, Jayachandran PT, Spanswick E, Danskin DW (2013a) GPS phase scintillation and proxy index at high latitudes during a moderate geomagnetic storm. Ann Geophys 31:805-816, doi:10.5194/angeo-31-805-2013

Prikryl P, Sreeja PV, Aquino M, Jayachandran PT (2013b) Probabilistic forecasting of ionospheric scintillation and GNSS receiver signal tracking performance at high latitudes. Spec Issue Ann Geophysics 56(2):R0222, doi:10.4401/ag-6219

Richardson IG (2013) Geomagnetic activity during the rising phase of solar cycle 24. J Space Weather Space Climate 3:A08, doi:10.1051/swsc/2013031

Richardson IG, Cane HV (2010) Near-earth interplanetary coronal mass ejections during solar cycle 23 (1996-2009): catalog and summary of properties. Solar Phys 264(1):189-237, doi:10.1007/s11207-010-9568-6

Spogli L, Alfonsi L, De Franceschi G, Romano V, Aquino MHO, Dodson A (2009) Climatology of GPS ionospheric scintillations over high and mid-latitude European regions. Ann Geophys 27:3429-3437

Sreeja V, Aquino M, Elmas ZG (2011) Impact of ionospheric scintillation on GNSS receiver tracking performance over Latin America: introducing the concept of tracking jitter variance maps. Space Weather 9:S10002, doi:10.1029/ $20115 W 000707$

van Dierendonck AJ, Arbesser-Rastburg B (2004) Measuring ionospheric scintillation in the equatorial region over Africa, including measurements from SBAS geostationary satellite signals. In: Proceedings of the 17th International Technical Meeting of the Satellite Division of The Institute of Navigation (ION GNSS 2004). The Institute of Navigation, Long Beach, CA, pp 316-324

Williams AO, Edberg NJT, Milan SE, Lester M, Fränz M, Davies JA (2011) Tracking corotating interaction regions from the Sun through to the orbit of Mars using ACE, MEX, VEX, and STEREO. J Geophys Res 116:A08103, doi:10.1029/ 2010JA015719

Wu C-C, Dryer M, Wu ST, Wood BE, Fry CD, Liou K, Plunkett S (2011) Global threedimensional simulation of the interplanetary evolution of the observed geoeffective coronal mass ejection during the epoch 1-4 August 2010. J Geophys Res 116:A12103, doi:10.1029/2011JA016947

Zhang X-Y, Moldwin MB, Cartwright M (2013) The geo-effectiveness of interplanetary small-scale magnetic fluxropes. J Atmos Solar-Terr Phys 95-96:1-14

Zheng Y, Macneice P, Odstrcil D, Mays ML, Rastaetter L, Pulkkinen A, Taktakishvili A, Hesse M, Kuznetsova MM, Lee H, Chulaki A (2013) Forecasting propagation and evolution of CMEs in an operational setting: what has been learned. Space Weather 11:557-574, doi:10.1002/swe.20096

\section{doi:10.1186/1880-5981-66-62}

Cite this article as: Prikryl et al: High-latitude GPS phase scintillation and cycle slips during high-speed solar wind streams and interplanetary coronal mass ejections: a superposed epoch analysis. Earth, Planets and Space 2014 66:62.

\section{Submit your manuscript to a SpringerOpen ${ }^{\odot}$ journal and benefit from:}

- Convenient online submission

- Rigorous peer review

- Immediate publication on acceptance

- Open access: articles freely available online

- High visibility within the field

- Retaining the copyright to your article

Submit your next manuscript at springeropen.com 